\title{
A method for optimal depth of the nasopharyngeal temperature probe: the philtrum to tragus distance
}

\author{
Hyungsun Lim, Jun Ho Lee, Kyung-keun Son, Young-Jin Han, and Seonghoon Ko \\ Department of Anesthesiology and Pain Medicine, Chonbuk National University Medical School and Hospital, Jeonju, Korea
}

Background: The nasopharyngeal temperature probe should be placed in the upper nasopharynx to reflect accurate core temperature. However, there have been no studies conducted to predict parameters for the optimal depth of the nasopharyngeal temperature probe. The purpose of this study was to examine the correlation between the optimal depth to the upper nasopharynx and the distance from the philtrum to the tragus and height.

Methods: Two hundred patients (100 females and 100 males) were enrolled in the study. The distance from the philtrum to the tragus along the facial curvature was measured, and the optimal depth from the nostril to the upper nasopharynx was evaluated using nasendoscopy. The relationships between the optimal depth to the upper nasopharynx and the distance from the philtrum to the tragus and height were examined.

Results: The distances from the philtrum to the tragus were $14.4 \pm 0.5 \mathrm{~cm}$ in females and $15.1 \pm 0.6 \mathrm{~cm}$ in males $(\mathrm{P}<0.01)$. The depths from the nostril to the upper nasopharynx were $9.4 \pm 0.6 \mathrm{~cm}$ in females and $10.0 \pm 0.5 \mathrm{~cm}$ in males $(\mathrm{P}<0.01)$. The correlation coefficients between the depth from the nostril to the upper nasopharynx and the distance to the tragus from the philtrum were 0.43 in females and 0.41 in males $(\mathrm{P}<0.01)$. However, there were very weak correlations and no correlations between height and the depth from the nostril to the upper nasopharynx in females and males, respectively. Conclusions: The depth from the nostril to the upper nasopharynx is correlated weakly with the distance from the philtrum to the tragus. Although the distance from the philtrum to the tragus is not a good predicting parameter for the optimal depth of nasopharyngeal temperature probe placement, subtraction of $5 \mathrm{~cm}$ from the distance is helpful to estimate the optimal depth of the nasopharyngeal temperature probe. (Korean J Anesthesiol 2014; 66: 195-198)

Key Words: Anesthesia, Nasopharynx, Temperature.

Received: August 27, 2013. Revised: 1st, September 13, 2013; 2nd, October 1, 2013. Accepted: October 4, 2013.

Corresponding author: Seonghoon Ko, M.D., Ph.D., Department of Anesthesiology and Pain Medicine, Chonbuk National University Hospital, 634-18, Keumam-dong, Jeonju 561-712, Korea. Tel: 82-63-250-1241, Fax: 82-63-250-1240, E-mail: shko@jbnu.ac.kr

(c) This is an open-access article distributed under the terms of the Creative Commons Attribution Non-Commercial License (http:// creativecommons.org/licenses/by-nc/3.0/), which permits unrestricted non-commercial use, distribution, and reproduction in any medium, provided the original work is properly cited. 


\section{Introduction}

Most anesthetic agents inhibit thermoregulatory function by interfering with hypothalamic reflex responses and additionally possess vasodilatory properties, which facilitate a flow of thermal energy from the core to the periphery of the body [1]. Because both the cold environment of the operating room and surgical exposure also contribute to excessive heat losses, inadvertent hypothermia is common during surgical procedures. Intraoperative hypothermia results in deleterious complications including myocardial ischemia, wound infection, coagulopathy, and delayed postanesthetic recovery [2-6]. Therefore, the core temperature should be monitored continuously for safe anesthesia [7].

Although the temperature measuring site varies according to the purpose of measurement, the nasopharynx is one of the most reliable sites for monitoring core temperature [8]. In addition, insertion of the temperature probe into the nasopharynx is easily performed by anesthetists. For these reasons, the nasopharynx is a commonly used temperature monitoring site during general anesthesia [9]. The nasopharyngeal temperature probe should be carefully placed at the upper or mid-portion of the nasopharynx to reflect accurate core temperature. However, there have been no studies conducted to predict parameters for the optimal depth of the nasopharyngeal temperature probe.

Because the tragus is located approximately $2 \mathrm{~cm}$ posterior to the posterior wall of the nasopharynx in the coronal plane of CT images, the authors hypothesized that the distance from the philtrum to the tragus might correlate with the depth from the nostril to the upper nasopharynx. The purpose of this study was to examine the correlation between the optimal depth to the upper nasopharynx and the distance from the philtrum to the tragus and height.

\section{Materials and Methods}

This study was approved by the Institutional Review Board and written informed consent was obtained from all participants. Two hundred elective surgical patients aged 18-75 years were enrolled. Patients were excluded if they had a history of nasal surgery, nasal trauma, or recurrent epistaxis. Other exclusion criteria included bleeding tendency or severe hepatic disease.

Patients did not receive preanesthetic medication and they did not receive vasoconstrictor nose spray. Patients were anesthetized with inhalational or total intravenous anesthetics. After induction of anesthesia, patients were maintained in a supine neutral position with a $5 \mathrm{~cm}$ pillow. The distance from the philtrum to the tragus was measured using a flexible ruler along the facial curvature. Because the upper or mid-portion of the nasopharynx is in close proximity to the internal carotid artery, the investigators measured the depth from the nostril to the upper nasopharynx using a nasendoscope (LF-GP, Olympus Medical System Corp., Tokyo, Japan) to determine the optimal depth for the nasopharyngeal temperature probe. The nasendoscope was advanced through the inferior meatus to reach the posterior upper nasopharyngeal mucosa, and the depth from the nostril to the upper nasopharynx was recorded. The authors divided the nasopharynx into an upper and a lower portion ad libitum. The correlation between the optimal depth to the upper nasopharynx and the distance from the philtrum to the tragus and height was evaluated.

\section{Statistical analysis}

Sample size was predetermined by the correlation sample size test using SigmaPlot 12.0 (Systat Software Inc., San Jose, CA, USA) based on the assumption that the correlation coefficient between the depth from the nostril to the upper nasopharynx and the distance from the philtrum to the tragus was 0.3 . It was ascertained that 85 patients were required with a significance level of $0.05(\alpha=0.05)$ and a desired power of $0.8(\beta=0.20)$. The sample size was increased to 100 for both genders.

Data are expressed as mean \pm SD. Data were analyzed using the t-test, Pearson correlation test, and linear regression. A P value less than 0.05 was considered statistically significant.

\section{Results}

Of the 200 enrolled surgical patients, no patient was excluded from data analysis. The mean ages of both genders were comparable (females: $50.6 \pm 11.8$ years, males: $50.9 \pm 12.7$ years, $\mathrm{P}=$ 0.42). Heights of the female and male patients were $156.0 \pm 6.9$ $\mathrm{cm}$ and $168.7 \pm 6.6 \mathrm{~cm}(\mathrm{P}<0.01)$, respectively. The distances from the philtrum to the tragus were $14.4 \pm 0.5 \mathrm{~cm}$ in females and $15.1 \pm 0.6 \mathrm{~cm}$ in males $(\mathrm{P}<0.01)$. The depths from the nostril to the upper nasopharynx were $9.4 \pm 0.6 \mathrm{~cm}$ in females and $10.0 \pm 0.5 \mathrm{~cm}$ in males $(\mathrm{P}<0.01)$.

The correlation coefficient between the depth to the upper nasopharynx $\left(D_{N P}\right)$ and the distance to the tragus from the philtrum $\left(\mathrm{T}_{\mathrm{P}-\mathrm{T}}\right)$ was 0.43 in females and 0.41 in males $(\mathrm{P}<0.01)$. The regression equations were $\mathrm{D}_{\mathrm{NP}}=2.35+\left(0.49 \times \mathrm{T}_{\mathrm{P}-\mathrm{T}}\right)$ and $\mathrm{D}_{\mathrm{NP}}=$ $4.71+\left(0.35 \times \mathrm{T}_{\mathrm{P}-\mathrm{T}}\right)$ in females and males, respectively (Fig. 1). There is a very weak correlation between the depth to the upper nasopharynx and height in females $(\mathrm{r}=0.27, \mathrm{P}<0.01$, ) but there is no correlation in males $(\mathrm{r}=0.14, \mathrm{P}=0.16)$.

\section{Discussion}

In the current study, anatomical factors were examined for a possible correlation to the optimal depth to appropriate naso- 
A

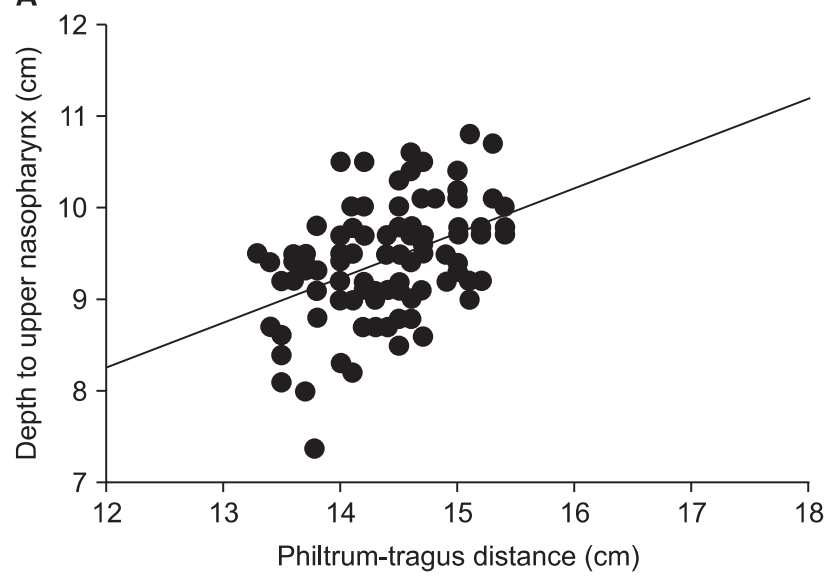

B

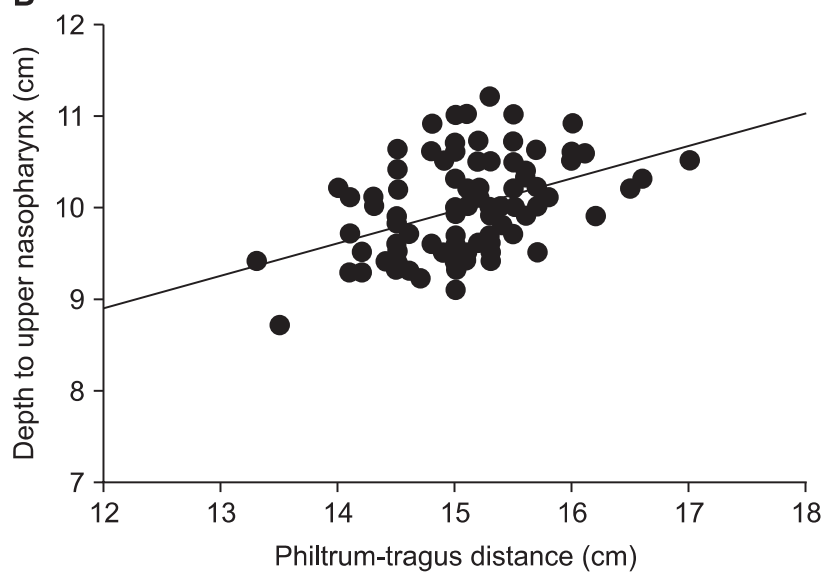

Fig. 1. There are significant correlations between the depth to the upper nasopharynx $\left(D_{N P}\right)$ and the distance to the tragus from the philtrum $\left(T_{P-T}\right)$ in females $\left(\mathrm{A}, \mathrm{r}=0.43, \mathrm{P}<0.01, \mathrm{D}_{\mathrm{NP}}=2.35+\left(0.49 \times \mathrm{T}_{\mathrm{P}-\mathrm{T}}\right)\right.$ and in males $\left(\mathrm{B}, \mathrm{r}=0.41, \mathrm{P}<0.01, \mathrm{D}_{\mathrm{NP}}=4.71+\left(0.35 \times \mathrm{T}_{\mathrm{P}-\mathrm{T}}\right)\right)$.

pharyngeal temperature probe placement. The distance from the philtrum to the tragus showed a correlation to the depth from the nostril to the upper portion of the nasopharynx to reflect accurate core temperature. However, there was no correlation between height and the depth from the nostril to the upper portion of the nasopharynx.

Measurement of body temperature is a standard component of perioperative patient monitoring to prevent deleterious physiologic effects of perioperative hypothermia including increased metabolic rate and cardiac work, decreased drug metabolism and cutaneous blood flow, and coagulopathy. The best temperature measurement sites for accurate monitoring are close to the central blood temperature which is considered the true core temperature. During general anesthesia the nasopharynx is commonly used to monitor temperature because it is one of the most reliable sites for measuring core temperature [9]. The optimal location for the nasopharyngeal temperature measurement, in close proximity to the internal carotid artery, is in the upper or mid-portion of the nasopharynx.

It is clinically difficult to use nasendoscopy to place the nasopharyngeal temperature probe at the optimal site. The authors attempted to find a simple anatomical predicting factor. Because the tragus is located approximately $2 \mathrm{~cm}$ posterior to the posterior wall of the nasopharynx in the coronal plane, the authors expected that the distance from the philtrum to the tragus would be correlated with the depth from the nostril to the upper nasopharynx.

The nasopharyngeal temperature probes can be inserted through the inferior or middle meatus in the nasal cavity. Because the probe path through the inferior meatus is straighter to reach the posterior nasopharyngeal wall, the depth from the nostril to the upper portion of the posterior nasopharyngeal wall through the inferior meatus is shorter than that through the middle meatus. Furthermore, when the nasopharyngeal temperature probe is advanced through the middle meatus, it is usually placed at the oropharynx. In this study, the nasendoscope was inserted through the inferior meatus. On the nasendoscopic measurement, the depths from the nostril to the upper portion of the nasopharynx through the inferior meatus were $9.4 \pm 0.6$ $\mathrm{cm}$ in females and $10.0 \pm 0.5 \mathrm{~cm}$ in males. There was a correlation between the depths from the nostril to the upper portion of the nasopharynx and the distance from the philtrum to the tragus. The distances from the philtrum to the tragus were 14.4 $\pm 0.5 \mathrm{~cm}$ and $15.1 \pm 0.6 \mathrm{~cm}$ in females and males, respectively. The difference between the distances from the philtrum to the tragus and the depths from the nostril to the upper nasopharynx was approximately $5 \mathrm{~cm}$ in both genders. Although our results showed that the regression equations between the depth to the upper nasopharynx $\left(\mathrm{D}_{\mathrm{NP}}\right)$ and the distance to the tragus from the philtrum $\left(\mathrm{T}_{\mathrm{P}-\mathrm{T}}\right)$ were $\mathrm{D}_{\mathrm{NP}}=2.35+\left(0.49 \times \mathrm{T}_{\mathrm{P}-\mathrm{T}}\right)$ in females and $\mathrm{D}_{\mathrm{NP}}=4.71+\left(0.35 \times \mathrm{T}_{\mathrm{P}-\mathrm{T}}\right)$ in males, these are complicated. Therefore, the authors recommend that the subtraction of $5 \mathrm{~cm}$ from the distance between the philtrum and the tragus is simpler and more useful in clinical practice. The authors expected that there would be a correlation between height and the depth from the nostril to the upper nasopharynx. There is a very weak correlation in female patients, but no correlation in male patients. Therefore, the authors could not recommend a heightbased formula.

The current study does have a limitation. The nasendoscope used in this study is more rigid than the commercial nasopharyngeal temperature probes. Because many commercial temperature probes can be bent in the nasal cavity, the actual distance to place the probe in the optimal site may be different from our recommendation. Furthermore, most commercial temperature probes have no length marker. This makes it difficult to know 
the inserted length of the probe. Therefore, using temperature probes which have a length marker could be helpful in placing the probe in the appropriate position.

In conclusion, the depth from the nostril to the upper nasopharynx is correlated weakly with the distance from the phil- trum to the tragus. Although the distance from the philtrum to the tragus is not a good predicting parameter for the optimal depth of nasopharyngeal temperature probe placement, subtraction of $5 \mathrm{~cm}$ from the distance is helpful to estimate the optimal depth of the nasopharyngeal temperature probe.

\section{References}

1. Butterworth JF, Mackey DC, Wasnick JD. Morgan \& Mikhail's Clinical anesthesiology. 5th ed. New York, McGraw-Hill Company. 2013, p 1184.

2. Kurz A, Sessler DI, Lenhardt R. Perioperative normothermia to reduce the incidence of surgical-wound infection and shorten hospitalization. Study of Wound Infection and Temperature Group. N Engl J Med 1996; 334: 1209-15.

3. Schmied H, Kurz A, Sessler DI, Kozek S, Reiter A. Mild hypothermia increases blood loss and transfusion requirements during total hip arthroplasty. Lancet 1996; 347: 289-92.

4. Lenhardt R, Marker E, Goll V, Tschernich H, Kurz A, Sessler DI, et al. Mild intraoperative hypothermia prolongs postanesthetic recovery. Anesthesiology 1997; 87: 1318-23.

5. Frank SM, Beattie C, Christopherson R, Norris EJ, Perler BA, Williams GM, et al. Unintentional hypothermia is associated with postoperative myocardial ischemia. The Perioperative Ischemia Randomized Anesthesia Trial Study Group. Anesthesiology 1993; 78: 46876.

6. Frank SM, Fleisher LA, Breslow MJ, Higgins MS, Olson KF, Kelly S, et al. Perioperative maintenance of normothermia reduces the incidence of morbid cardiac events. A randomized clinical trial. JAMA 1997; 277: 1127-34.

7. Sessler DI. Temperature regulation and monitoring. In: Miller's Anesthesia. 7th ed. Edited by Miller RD: Philadelphia, Churchill Livingstone. 2010, pp 1551-2.

8. Stone JG, Young WL, Smith CR, Solomon RA, Wald A, Ostapkovich N, et al. Do standard monitoring sites reflect true brain temperature when profound hypothermia is rapidly induced and reversed? Anesthesiology 1995; 82: 344-51.

9. Torossian A. Survey on intraoperative temperature management in Europe. Eur J Anaesthesiol 2007; 24: 668-75. 